\title{
Hommage
}

\section{Daniel Blanc nous a quittés}

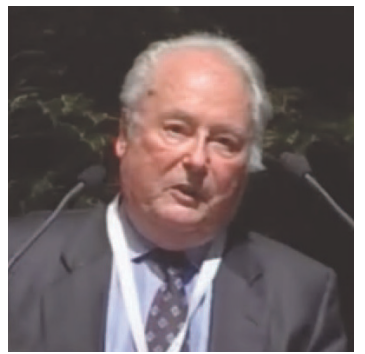

À quelques jours de son $82^{\mathrm{e}}$ anniversaire, Daniel Blanc nous a quittés le 5 novembre 2009, victime d'une terrible maladie, contre laquelle il avait pourtant consacré une très grande part de sa carrière.

Beaucoup d'entre nous sont accablés par cette triste nouvelle, car Daniel a été pour la Société française de radioprotection un modèle, tant il s'est investi dans notre société tout au long de sa vie.

Rappelons tout d'abord qu'il a participé à la création de la revue Radioprotection, qu'il a présidé la commission de la revue de 1969 à 1973, puis de juin 1987 à 1996, année où il m'a demandé de lui succéder. Il a été également président de notre société de juin 1968 à juin 1969. À cette époque, les présidences ne duraient qu'un an. C'est lui qui a créé le « Club histoire », qu'il a présidé de 1997 à 2005 avant que Jacques Lochard ne lui succède. Un tel engagement dans une société, la nôtre, est unique et aujourd'hui bon nombre d'entre nous se sentent orphelins car pour tous Daniel est un modèle et un ami.

Il aimait rappeler que, venant de Caen, le professeur Rocard, lui avait demandé de créer un enseignement de physique atomique à Toulouse. À ses débuts, en janvier 1957, le laboratoire fut nommé «Laboratoire de physique corpusculaire de Toulouse » (LPCT), mais très vite son nom changea pour mieux correspondre à ses missions et, en 1958, ce fut la création du Centre de physique nucléaire (CPNT). Nouvelle orientation en 1967, où ce centre devient le centre de physique atomique et nucléaire de Toulouse (CPANT) avant de devenir en 1972 le Centre de physique atomique de Toulouse, le CPAT. En 1984, quelques changements surviennent encore, mais Daniel Blanc reste Directeur du CPAT et responsable de la section Physique radiologique jusqu'en 1987, lorsque Michel Terrissol lui succède. En 1995, le sigle reste, mais sa signification change, pour devenir le centre de physique des plasmas et leurs applications de Toulouse, qui jouit d'une belle renommée internationale.

Au tout début Daniel rappelait que les intentions de l'université de Toulouse étaient « floues » et que l'argent manquait, et il lança le laboratoire dans le domaine de l'électronique nucléaire dans lequel il avait réalisé sa thèse de Docteur ès sciences pour avancer en terrain connu. Les axes de recherches furent donc la détection. Ce laboratoire s'installa alors sur le campus universitaire de Rangueil.

Andrée Dutreix nous rappelle que le premier poste de radiophysicien fut créé en 1953 à l'Institut Gustave Roussy. Ils n'étaient que trois spécialistes en France

DOI: $10.1051 /$ radiopro/2009028

RADIOPROTECTION - VOL. 44 - N 4 (2009)

Article published by EDP Sciences and available at http://www.radioprotection.org or http://dx.doi.org/10.1051/radiopro/2009028 
en 1963, José Blocquel à Caen et Jean Chavaudra à l'IGR. Andrée Dutreix a puisé en permanence dans le vivier des jeunes chercheurs du laboratoire de Daniel Blanc qui lui a suggéré de créer un DEA. Elle crut alors... rêver! Vous imaginez facilement tous les obstacles qu'il a fallu franchir pour créer un DEA à cheval sur deux universités Paris et Toulouse, et sur deux disciplines, la physique théorique et les applications médicales. Mais l'opiniâtreté de Daniel Blanc a eu raison de toutes les réticences et il fut, jusqu'au bout, un inlassable défenseur de cette formation. L'histoire de cette aventure de 1970 à 1996, peut être consultée in extenso sur le site de notre société (www.sfrp.asso.fr). Nous y trouvons avec plaisir dans la première promotion - et un brin de nostalgie... - les noms de notre trésorier Bernard Aubert et deux de nos administrateurs, Alain Biau aujourd'hui, Hélène Aget il y a quelques années. Plus tard, Josette Champlong, présidente de la section PCR fut également une élève de Daniel Blanc. J'arrêterai là les citations tant la liste des promus est riche et Bernard Aubert apporte ici le témoignage d'un ancien élève, à la fois membre de la SFRP et de la Société française de physique médicale (SFPM).

Mais là ne s'est pas arrêté le dynamisme de Daniel Blanc. Dès les années 1960, une première association fut créée entre le laboratoire de dosimétrie de Fontenayaux-Roses et l'université Paul Sabatier de Toulouse. En 1985, ces deux pôles ont conscience de l'importance de fédérer tous les laboratoires universitaires travaillant dans le domaine de la dosimétrie dans une structure associative de type 1901, afin de mieux coordonner leurs travaux et surtout de leur donner une véritable lisibilité au niveau de la Commission des communautés européennes. Avec humour, cette association, cofondée par Daniel Blanc et Gui Portal s'intitule «les LARD» (Laboratoires associés de radiophysique et de dosimétrie) et Daniel n'a jamais pu s'empêcher d'appeler ses membres, de façon hypocoristique, ses « lardons ! ». Les témoignages du cofondateur et du président actuel des LARD sont parlants.

Daniel aimait transmettre son savoir et il laisse une série de livres qui furent tous rééditées. Je n'en citerai que quelques uns: Les rayonnements ionisantsdétection, dosimétrie, spectrométrie (Masson, 1990-1997), Physique nucléaire (Masson, collection abrégés, 1993) Physique nucléaire, particules (Masson, 1995), Physique nucléaire pour les médecins et biologistes (Masson, collection abrégés, 1993, 1995), mais aussi Fusion thermonucléaire contrôlée, une énergie pour le futur en 1978.

Daniel, le scientifique reconnu par tous ses pairs, était aussi un artiste, féru de Jazz. Il collectionnait une série impressionnante d'enregistrements et je me rappelle qu'un jour, en examinant ma modeste «jazzothèque », il me prodigua quelques conseils pour la rendre plus éclectique. Après Antibes ou Nice, où il commença ses travaux pratiques, Daniel fut un fidèle spectateur du festival de Marciac, à une époque où celui-ci n'avait pas encore trouvé ses lettres de noblesse. Gastronomie 
et jazz, il ne pouvait manquer un tel rendez-vous ! Mais là ne s'arrêtait pas son immense culture. L'Académie des sciences, inscriptions et Belles Lettres de Toulouse en témoigne.

Et dans ce trop bref hommage que notre société rend à Daniel Blanc, je voudrais dire combien j'ai été heureux et honoré lorsqu'il me demanda, en 1993, de devenir vice-président du comité de rédaction, et, ce, d'autant plus qu'il avait assorti cette nomination d'un « droit de succession », lorsqu'il le souhaiterait, i.e. en 1996. Nous avons voulu à l'époque donner un nouveau souffle à la revue, élargir la représentativité du comité, ouvrir nos colonnes à des auteurs étrangers. Nous avons également lutté pour faire admettre l'anglais dans notre revue. Nous avons voulu montrer que la revue n'appartenait pas à un cénacle parisien, en lançant les réunions délocalisées, dont la première s'est tenu à Golfech en 1994. Nous devenions «VRP» de notre revue... Jusqu'au bout ou presque, Daniel a participé à nos réunions, apportant toujours sa grande sagesse à nos débats, pas un de mes éditoriaux mis à part ce dernier, ne fut relu et annoté par Daniel. C'est dire, toute la tristesse que nous ressentons au comité de rédaction, et avec Jeannine Lallemand, notre vice présidente, Jacques Lombard, notre secrétaire général, Agnès Henri notre éditrice et tous les membres du comité nous voulons dire une nouvelle fois $\mathrm{Merci}$ Daniel et transmettre à Françoise, son épouse, ainsi qu'à ses enfants et petitsenfants, toute notre sympathie, en espérant que l'estime, l'admiration et le souvenir inaltérable que tous ses amis lui portent, leur soient réconfort. Nous sommes sûrs que, depuis le Panthéon de l'Harmonie, devenu désormais sa résidence principale, Daniel continuera à nous éblouir et à nous interpeller. Sa belle lumière restera présente dans nos pensées, puisque nous savons tous que «le vrai tombeau des morts, c'est le cœur des vivants » (Tacite).

Henri Métivier

Président du comité de rédaction Radioprotection, Revue de la Société française de radioprotection

***

À quelques mois de célébrer les 40 ans de la création de la formation en physique médicale, le professeur Daniel Blanc nous a quittés. Cette nouvelle m'a profondément affecté car il a accompagné toute ma carrière professionnelle. C'est durant l'année universitaire 1969-1970 que je l'ai rencontré pour la première fois en tant que responsable du C3 de «Physique atomique et nucléaire » que je suivais dans le cadre de la maitrise de physique-chimie. Il sut convaincre un petit noyau d'étudiants de le suivre dans l'aventure d'une nouvelle formation: le DEA de physique atomique, option « Physique radiologique». 
Par la suite, j'ai toujours gardé le contact, d'abord dans le cadre de ma thèse, puis quelques années plus tard, en partageant la responsabilité de la formation à l'Institut Gustave Roussy avec Jean Chavaudra. Ces années là nous amenaient à nous rendre régulièrement à Toulouse pour la sélection des étudiants, les examens du DEA et les soutenances de stage. Elles m'ont permis d'apprécier, non seulement la rigueur et l'exigence de Daniel Blanc vis-à-vis de ses étudiants, mais également son souci permanent de les aider à mener à bien leur projet professionnel, ainsi que son enthousiasme, sa convivialité et son sens de l'hospitalité.

Daniel Blanc a également joué un rôle important dans ma carrière, tout d'abord en insistant, avec l'appui d'Andrée et Jean Dutreix, pour que certains radiophysiciens soutiennent leur habilitation à diriger des recherches. C'est également lui qui m'a orienté vers la SFRP et l'IPSN, ce qui n'est sûrement pas étranger à ma situation actuelle.

Pour toutes ces raisons Daniel Blanc restera pour moi l'une des personnes pour qui j'ai le plus grand respect et dont la disparition m'a profondément marqué. Il restera à jamais l'un des fondateurs de la formation en physique médicale avec près de 400 physiciens formés de 1970 à 1995. Il manquera à tous ceux qui l'ont connu et qui le retrouvaient toujours avec grand plaisir lors des congrès de la SFPM ou de la SFRP.

Bernard Aubert

Trésorier de la SFRP

Membre de la Société française de physique médicale

$* * *$

\section{Le professeur Daniel Blanc et les LARD}

Le laboratoire de recherches en dosimétrie des rayonnements que je dirigeais au CEA de Fontenay-aux-Roses s'était associé au Centre de physique atomique de Toulouse (CPAT) dont le directeur, le professeur Daniel Blanc, était mon conseiller scientifique. Le laboratoire du CEA bénéficiait ainsi d'une structure universitaire capable d'apporter le soutien de chercheurs dont les connaissances en physique fondamentales étaient essentielles pour le développement de techniques dosimétriques nouvelles.

Au fil des années le domaine de recherches en dosimétrie des rayonnements s'étant élargi, Daniel m'a aidé à nouer progressivement des contacts avec divers laboratoires universitaires français et étrangers. Ces relations étant initialement unilatérales, Daniel m'a fait remarquer qu'il fallait créer une structure de coordination pour faciliter la rencontre des chercheurs, définir les objectifs de 
recherche et répartir les études. C'est l'origine de la création des LARD (Laboratoires associés de radiophysique et de dosimétrie) crées en 1985.

C'est ainsi que se sont regroupées, dès l'origine, les universités de Nice (Laboratoire d'émission électronique), de Limoges (Laboratoire d'électronique des polymères sous faisceau ioniques), de Montpellier (université des sciences et techniques de Languedoc), de Saint-Étienne (laboratoire de physique des processus industriels de l'École des mines), ainsi que le centre de recherches nucléaires de Kronenbourg du CNRS et le Laboratoire de biophysique des rayonnements de l'INSERM de Strasbourg. Deux laboratoires étrangers représentant les universités de Prague et de Barcelone s'y sont également associés. Aujourd'hui, les LARD sont constitués de 16 laboratoires.

La commission des communautés européennes qui, à l'origine, ne soutenait financièrement que le laboratoire du CEA, a ainsi été amenée à élargir son champ d'action à l'ensemble des laboratoires des LARD. Les recherches effectuées en France ont alors pris une importance qui a été rapidement reconnue par les laboratoires européens impliqués dans le même domaine de recherche. Ceux-ci, ne bénéficiant pas d'une structure nationale équivalente, ont laissé à la France le « leadership » dans bien des secteurs de recherche.

Les présidents successifs et les membres des LARD ont progressivement développé cette prééminence. Didier Paul, le président actuel qui a infléchi l'action des LARD en l'ouvrant aux étudiants qu'il encourage et récompense, ne démentira pas cette évidence. Ils ont hérité du génie de Daniel qui leur a montré la voie de l'entraide et de la coordination.

Merci Daniel! Tu as fait de très grandes choses mais cela ne doit pas nous étonner car tu as été un être « tout à fait exceptionnel».

Gui Portal

Cofondateur des LARD

Daniel Blanc et Gui Portal co-fondateurs des LARD sont toujours restés fidèles à leur création, jusqu'au jour où Daniel nous quitte laissant derrière lui des orphelins

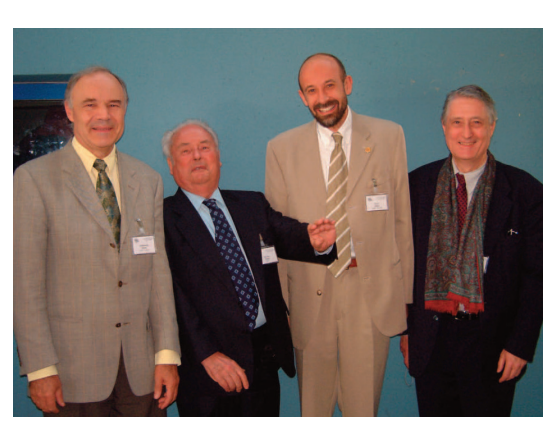
de la physique radiologique et médicale qui viennent de perdre l'un de leur pères, leur Professeur, président du jury de thèse ou encore directeur de thèse de doctorat. Ses ouvrages nous ont tous instruits et prolongeront ainsi son œuvre immense pour les nouveaux étudiants qui nous rejoignent et les enseignants dont je fais partie. J'ai eu aussi la lourde tâche de prendre après d'autres collègues formés 
HOMMAGE

à ses côtés, la présidence des LARD. Son départ nous a tous bouleversés et au nom des LARD, j'exprime toute notre reconnaissance et nos remerciements pour celui qui a su nous rassembler et faire progresser, connaitre nos recherches dans la convivialité et l'excellence. Parmi ses derniers écrits, il nous a fait l'honneur de préfacer le numéro spécial de Radioprotection consacré aux LARD pour leur vingtième anniversaire.

Merci infiniment Daniel, l'infini existe en physique...

Didier Paul

Président des LARD 